\title{
Research on English language teaching evaluation system based on big data technology
}

\author{
Chu Weili \\ Qingdao Huanghai University, Qingdao, Shandong, China
}

\begin{abstract}
In this paper the standard of English teaching evaluation and establishes an effective teaching evaluation system was analyzed based on big data technology. It is proved by experiment that this method has a good effect on the evaluation of English teaching effect, and has expanded the new development direction for modern English teaching.
\end{abstract}

Keywords: English language teaching, Quality assessment system, Data mining.

\section{Introduction}

To monitor college's English Teaching quality, online evaluating the teacher's in-class English Teaching skill has become an important way. In each year, dean's office requires students to grade the teacher's English Teaching skill by logging in English Teaching evaluation model of educational management system. Thus, the system has accumulated a large number of assessment data from students. Conventional methods used to analyze these data are simple and statistical concept. Also, the deep association analysis is not made to mine the various factors of impacting the English Teaching quality evaluation results. It is an urgent task to provide decision support for the administrator by effectively analyzing and processing these data so as to further mine the valuable hidden information.

Data mining (DM) technique is an effective way to acquire valuable information from mass data and has been widely applied to all kinds of fields [1,2] such as in telecommunication, retail sale, insurance, and finance since these fields usually accumulate a large amount of data. $\mathrm{DM}$, also known as knowledge discover in database (KDD), is currently hot topic in artificial intelligence and database area $[3,4]$. With help of DM technique, analytical deduction, mining potential pattern, adjusting marketplace strategy, decreasing risks and true decision can be made effectively for policymakers. Association rule is a DM method which can discover the hidden correlation in data. In general, some rules satisfied with certain conditions can always be found in large-scale database. But, only the strong rules are meaningful and valuable for user. Thus, two thresholds, namely support and confidence, are usually adopted to delete some useless rules. In other word, if the support of a rule is very low, this rule will just appear occasionally. The confidence reflects the reliability of the rule. Only the rules whose confidences are larger than a certain threshold are reliable. The user employs these two thresholds to further refine the mined rules. The commonly used association rule algorithms include Apriori, FP-Growth, etc. Apriori is the most typical algorithm [5]. Based on a priori knowledge of frequent itemsets, Apriori adopts a layer-by-layer iteration searching. In Apriori algorithm, all frequent 1 itemsets are first searched by scanning the transaction records. All of the frequent 1 itemsets are denoted as the set L1. Then, L1 is used to search the set L2 of frequent 2 itemsets, and L2 serves as L3 in the same way. The iteration continues until no frequent $\mathrm{K}$ itemset is found. Finally, the strong rules can be extracted from all the frequent itemsets. These strong association rules are interest for user [6]. Decision tree algorithm is a typical classification method. C4.5 is the most commonly used decision tree algorithm. It adopts information gain ratio [7] to overcome the drawback of tending to select the attributions with many values when only using information gain in ID3 [8]. Using C4.5, the continuous attribution can be discretized and the incomplete data can be also processed. Up to now, many English Teaching quality evaluation systems based on DM have also been developed. However, there are few published works focusing on analyzing the evaluation 
results. Besides, the practical applications of the obtained rules are not well investigated in literature.

In order to sufficiently utilize the mass data that the educational management system have accumulated over the years and discover the inherent rule and knowledge hidden in these data, this paper focuses on investigating DM technique and its application in digital campus educational management system. To be specific, we adopt the classification technique and the association rule to analyze the evaluation data on teacher's English Teaching quality. Then, we exploit $\mathrm{C} 4.5$ to construct the decision tree of course' characters, which can be used to mine the potential relationship between the course' characters and the English Teaching quality. Finally, we further employ Aprior algorithm to discover some important rules between the teacher's features and his in-class English Teaching quality results. In a consequence, we expect that our mined rules can provide great help for the educational policymaker.

\section{Case Analysis}

1) Applying association analysis to mine rules between the teacher's attribution and the English Teaching quality evaluation result

This section focuses on exploring the relationship between the evaluation result and the teacher's attribution including title, degree, age, seniority, and load. We select the teacher's attribute and the English Teaching evaluation results from the first semester of 2012 to 2013 year as the test dataset. Part of them is shown as Table 1. It is clear that the data format in Table 1 cannot be directly used to perform Apriori algorithm. They must be converted into transaction database. In conversion, all of the attribution values are first discretized to the corresponding code formats. The codes of various attribution values are as shown in Table 2 . Each row in Table 1 is acted as a transaction record. Every field of a record is converted to the corresponding item using codes table (Please refer to Table 2.). For instance, a record is as follows:

$$
\begin{array}{llllllll}
\text { ID } & \text { Age } & \text { Sex } & \text { Degree } & \text { Title } & \text { Seniority } & \text { Load } & \text { Grade } \\
103215 & 38 & \text { Male } & \text { Doctor } & \text { Associate professor } & 7 & 241.68 & \text { Good }
\end{array}
$$

This record is then converted to the corresponding transaction as follows:

$\{\mathrm{A} 16, \mathrm{~A} 1, \mathrm{~A} 5, \mathrm{~A} 7, \mathrm{~A} 9, \mathrm{~A} 21, \mathrm{~A} 23\}$

TABLE 1. THE TEST DATASET ABOUT TEACHER'S ATTRIBUTION AND EVALUATION RESULT.

\begin{tabular}{llllllll}
\hline ID & Age & Sex & Degree & Title & Seniority(Years) & Load(Hours) & Grade \\
\hline 100033 & 40 & Female & Doctor & Associate professor & 19 & 44 & Excellent \\
100047 & 50 & Male & Master & Professor & 21 & 64.2 & Excellent \\
100048 & 47 & Male & Master & Associate professor & 15 & 123.2 & Excellent \\
100056 & 43 & Male & Master & Associate professor & 20 & 220 & Excellent \\
100057 & 45 & Female & Master & Professor & 24 & 69.2 & Excellent \\
103373 & 34 & Male & Doctor & Lecturer & 2 & 193.1 & Good \\
103397 & 32 & Male & Doctor & Lecturer & 2 & 216.4 & Good \\
103215 & 38 & Male & Doctor & Lecturer & 5 & 30 & Good \\
103357 & 26 & Female & Master & Lecturer & 2 & 292 & Excellent \\
103407 & 40 & Male & Doctor & Lecturer & 2 & 147.39 & Excellent \\
103444 & 36 & Male & Doctor & Associate professor & 7 & 241.68 & Good \\
$\ldots \ldots \ldots \ldots$. & $\ldots \ldots$ & $\ldots \ldots$ & $\ldots \ldots$ & $\ldots \ldots$. & $\ldots \ldots \ldots$ & $\ldots \ldots \ldots .$. & $\ldots \ldots \ldots$ \\
\hline
\end{tabular}

\begin{tabular}{|c|c|c|c|c|c|}
\hline Attribution & Value & Code & Attribution & Value & Code \\
\hline \multirow[t]{2}{*}{ Sex } & Male & A1 & \multirow[t]{2}{*}{ Load } & $0 \sim 120$ & A20 \\
\hline & Female & $\mathrm{A} 2$ & & Larger than 120 & A21 \\
\hline \multirow{3}{*}{ Degree } & Bachelor & A3 & \multirow{3}{*}{ Title } & Lecturer & A6 \\
\hline & Master & A4 & & Associate prof. & A7 \\
\hline & Doctor & A5 & & Professor & $\mathrm{A} 8$ \\
\hline \multirow{4}{*}{ Seniority } & $1 \sim 10$ & A9 & \multirow{4}{*}{ Age } & $0 \sim 35$ & A15 \\
\hline & $11 \sim 20$ & $\mathrm{~A} 11$ & & $36 \sim 40$ & A16 \\
\hline & $21 \sim 30$ & A13 & & $41 \sim 45$ & A17 \\
\hline & More than 30 & A14 & & $46 \sim 50$ & A18 \\
\hline
\end{tabular}

TABLE 2. ATTRIBUTION VALUES AND THEIR CODES. 


\begin{tabular}{|l|l|l|l|l|l|}
\hline \multirow{4}{*}{ Grade } & Excellent & A22 & & $50 \sim 65$ & A19 \\
\cline { 2 - 3 } & Good & A23 & & & \\
\cline { 2 - 3 } & Average & A24 & & & \\
\cline { 2 - 3 } & Pass & A25 & & & \\
\hline
\end{tabular}

After processed in the above, we can finally obtain a transaction database about the in-class English Teaching evaluation result as shown in Table 3. The data after being preprocessed is considered to be relatively pure. Then, we perform Apriori algorithm on the preprocessed data. Apriori algorithm resorts to spss Clementine tool. In the experiment, the support, the confidence, and the largest number of itemsets are set to be $0.2,0.7$, and 5 , respectively. The final mined association rules consist of 40 items, part of which is shown in Table 4.

We can see from Table 4 that according to the rule 3 the team which consists of young and middle-aged teachers with associate professor and doctor degree play an important role in English Teaching staff. Therefore, these teachers should be placed on the English Teaching front line so that they can play a vanguard and exemplary role in English Teaching. It can still be observed that the teachers with age of lower than 50, associate professor and master degree have very rich English Teaching experience. The young teachers can ally with the old teachers so as to learn from them. In so doing, the English Teaching ability of the young teachers can be improved fast and fully. In recruit, the young doctors and the experienced teachers with title of professor should be superiorly considered so as to improve the level of the whole teacher team

TABLE 3. TRANSACTION DATABASE ABOUT TEACHER'S ATTRIBUTION AND EVALUATION RESULT.

\begin{tabular}{|c|c|c|c|c|c|c|c|}
\hline ID & Age & Sex & Degree & Title & Seniority & Load & Grade \\
\hline 100033 & A16 & $\mathrm{A} 2$ & A5 & A7 & A11 & A20 & A22 \\
\hline 100047 & A18 & A1 & A4 & A8 & A13 & A20 & A22 \\
\hline 100048 & A17 & A1 & A4 & A7 & A13 & A21 & A22 \\
\hline 100056 & A17 & A1 & A4 & A7 & A11 & A21 & A22 \\
\hline 100057 & A14 & $\mathrm{A} 2$ & A4 & A8 & A13 & A20 & A22 \\
\hline 103373 & A15 & A1 & A5 & A6 & A9 & A21 & A23 \\
\hline 103397 & A15 & Al & A5 & A6 & A9 & A21 & A23 \\
\hline 103215 & A15 & A1 & A5 & A6 & A9 & A20 & A23 \\
\hline 103357 & A15 & $\mathrm{A} 2$ & A4 & A6 & A9 & A21 & A22 \\
\hline 103407 & A15 & A1 & A5 & A6 & A9 & A21 & A22 \\
\hline 103444 & A15 & A1 & A5 & A7 & A9 & A21 & A23 \\
\hline ...m.w. & $\ldots \ldots$ & $\ldots \ldots$ & $\ldots \ldots$ & $\ldots \ldots$ & ....... & ......... & ....... \\
\hline
\end{tabular}

TABLE 4. THE OBTAIN RULES UNDER SUPPORT OF 0.2 AND CONFIDENCE OF 0.7 CONDITIONS.

\begin{tabular}{|c|c|c|c|}
\hline Input & Result & Support & confidence \\
\hline Degree $=$ Master, Title $=$ Associate prof., Age $<=50$ & Excellent & $20.14 \%$ & $87.3 \%$ \\
\hline Seniority $>=10$, Degree $=$ Master & Excellent & $20.25 \%$ & $76.8 \%$ \\
\hline Degree $=$ Doctor, Title $=$ Associate prof. & Excellent & $21.6 \%$ & $95.6 \%$ \\
\hline Degree $=$ Master, Title $=$ Lecturer, Load $>=120$ & Excellent & $20.52 \%$ & $72.65 \%$ \\
\hline Degree $=$ Master, Title $=$ Lecturer, Load $>=120$ & Excellent & $22.85 \%$ & $80.35 \%$ \\
\hline & $\ldots \ldots$ & $\ldots \ldots \ldots$ & $\ldots \ldots \ldots \ldots$ \\
\hline
\end{tabular}

2) Applying decision tree to mine rules between the course's attributions and English Teaching quality evaluation results

Apart from the teacher's attribution, the course's attribution, such as credit, week hour, property, examination style, and numbers, may have a certain effect on the English Teaching quality evaluation results. This section aims at mining the hidden rule about how the course's attribution impacts the English Teaching quality evaluation results. We take the English Teaching task and English Teaching evaluation data in the first semester of 2012 to 2013 as the test data. This test data includes 3000 records. Part of them is as shown in Table 5. 
TABLE 5. THE TEST DATASET ABOUT COURSE'S ATTRIBUTION AND EVALUATION RESULT.

\begin{tabular}{lllllll}
\hline ID & Credit & Week hour & Property & Style & Numbers & Grade \\
\hline 100033 & 2 & 36 & Required & Exam & 26 & Excellent \\
100047 & 2 & 36 & Selective & Check & 27 & Excellent \\
100048 & 1.5 & 54 & Basic & Check & 27 & Excellent \\
100057 & 2 & 36 & Selective & Exam & 27 & Excellent \\
103373 & 3 & 54 & Basic & Exam & 40 & Good \\
103215 & 2 & 36 & Selective & Check & 43 & Good \\
103357 & 1 & 28 & Required & Exam & 27 & Good \\
103407 & 3.5 & 72 & Basic & Check & 35 & Excellent \\
103444 & 4 & 72 & Required & Exam & 37 & Excellent \\
$\ldots \ldots \ldots \ldots .$. & $\ldots \ldots$ & $\ldots \ldots$. & $\ldots \ldots \ldots \ldots \ldots$ & $\ldots \ldots \ldots$ & $\ldots \ldots$ & $\ldots \ldots$ \\
\hline
\end{tabular}

We choose TipDM tool [9] to construct DM model. C4.5 decision tree module of TipDM is used for evaluating the test 3000 records. In experiments, the target decision tree is finally obtained through data inputting, parameters set, and model training. We extract the part of the rules as follows:

Rule 1: course property =basic course and week hour $>22$ THEN the ratio of excellent is $31.3 \%$.

Rule 2: course property $=$ required course and examination style $=$ check and score $<=1.5$ and week hour $<=24$ THEN the ratio of excellent is $21 \%$.

Rule 3: course property $=$ selective course and week hour $<=45$ and numbers $<=42$ THEN the ratio of excellent is $33.8 \%$.

It can be observed from our mined rules that there is not necessary connection between the number of students and the evaluation grades. However, these courses with 22-45 week hours obtain relatively better evaluations. This is because the week hours of these courses are moderate and perfectly match the tolerable force of the student. We can still see that the basic and required courses have high grade. It is mainly due to the facts that these courses are professional and their credits are high. Naturally, these courses are very crucial for students, thus resulting in better evaluation.

\section{Conclusions}

The in-class English Teaching quality evaluation is an important way to assess the teacher's English Teaching skill thus becoming a vital part of the higher education management. This paper attempts to apply data mining technique to discover some hidden rules and valuable information. The English Teaching quality evaluation data accumulated over the years are selected as the test data. We create the two DM models, which are about how the teacher's attribution and the course's attribution affect the evaluation results, respectively. In contrast, the two DM algorithms, namely association analysis and decision tree, are respectively used to analyze the corresponding DM model. The experimental results show that our mined rules are reliable and practical. Therefore, these rules can significantly improve the efficiency of policy maker of higher education system.

\section{References}

[1] M. Chen, J. Han, P. Yu, "Data Mining: An overview from database perspective," IEEE Transactions on knowledge and Data Engineering, vol.8, no.6, pp.866-883, 1996.

[2] J. Han, M. Kamber, Data Mining Concepts and Techniques, San Francisco: Morgan Kaufmann Publishers, 2005. 
[3] J. E. Beck, "Engagement tracing: using response times to model student disengagement", In Proceedings of the 12th International Conference on Artificial Intelligence in Education, pp.88-95, 2005.

[4] F. Usama, P. Gregory, P. Smyth, "From data mining to knowledge discovery in databases," $A I$ Magazine, vol.17, no.3, pp.23-34, 1996.

[5] R. Agrawal, T. Imielinski, A. Swami, "Mining association rules between sets of items in large databases," In Proceedings of the ACM SIGMOD Conference on Management of Data, pp.207-216, 1993. 